\title{
Prueba de la conductividad eléctrica en la evaluación fisiológica de la calidad de semillas en Zeyheria tuberculosa
}

\author{
Electrical conductivity test in the evaluation of physiological quality in seeds of \\ Zeyheria tuberculosa
}

\author{
José Luis Soto Gonzales a*, Sérgio Valiengo Valeri ${ }^{\text {b }}$ \\ *Autor de correspondencia: ${ }^{a}$ Universidad Estadual Paulista, UNESP-FCAV, Jaboticabal, San Pablo, \\ Brasil, 14884-900. Tel. 11-96184922, jolusogol@hotmail.com

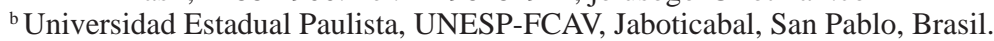

\begin{abstract}
SUMMARY
The electrical conductivity test is still an excellent tool to evaluate the effect of seeds of various forest and agricultural species and recent studies have been conducted aiming at verifying its application in forest seeds. The objective of this study was to establish a specific methodology to test the electrical conductivity of forest seeds of Zeyheria tuberculosis. Four lots of seeds were used, which were submitted to the germination test, evaluating the percentage of germination, germination speed index and dry mass of seedlings. For the electrical conductivity test, five replicates of 20 seeds installed in three volumes of deionized water $(75,100$ and $125 \mathrm{~mL})$ were used and eight periods for seed imbibitions $(2,4,6,12,18,24,48$ and 72 hour $)$ at $25^{\circ} \mathrm{C}$ were allowed. The statistical design used was completely random; the comparison of means was performed by Tukey test at $5 \%$ probability. Lot II showed higher germination percentage and speed. The electrical conductivity test allows discrimination of the same batch by the germination test under laboratory conditions. It was possible to separate the seed lot presenting better physiological quality (lot II) from among the other lots. It was recommended the use of 75 or $125 \mathrm{~mL}$ of deionized water at a temperature of $25^{\circ} \mathrm{C}$ to perform the electrical conductivity test.
\end{abstract}

Key words: vigor of seeds, native forest seed, seed physiological quality.

\section{RESUMEN}

La prueba de la conductividad eléctrica de las semillas es una excelente herramienta para evaluar el vigor de semillas de diversas especies forestales y agrícolas. Recientemente la prueba ha sido aplicada en semillas forestales. El objetivo del presente trabajo fue adaptar dicha metodología para realizar la prueba de conductividad eléctrica y estudiar el volumen de agua y el periodo de imbibición para evaluar la calidad fisiológica de lotes semillas forestales de Zeyheria tuberculosa. Cuatro lotes de semillas (árboles madre) fueron sometidos a la prueba de germinación para evaluar el porcentaje de germinación, el índice de velocidad de germinación y materia seca de plántulas. Para la prueba de conductividad eléctrica fueron utilizados cinco repeticiones de 20 semillas cada una, instaladas en tres volúmenes de agua desionizada $(75,100$ y $125 \mathrm{~mL})$, con ocho periodos de imbibición $(2,4,6,12,18,24,48$ y 72 horas) a $25^{\circ} \mathrm{C}$. El diseño estadístico empleado fue completamente al azar y la prueba usada fue Tukey al $5 \%$ de propabilidad. El lote II presentó mayor porcentaje y velocidad de germinación. La prueba de la conductividad eléctrica y de germinación juzgaron igual a los lotes de semillas. Se recomienda el uso de 75 o $125 \mathrm{~mL}$ de agua desionizada, a la temperatura de $25^{\circ} \mathrm{C}$, para la realización de la prueba de la conductividad eléctrica.

Palabras clave: vigor de semillas, semilla forestal nativa, calidad fisiológica de semillas.

\section{INTRODUCCIÓN}

Zeyheria tuberculosa Vell. Bur (familia Bignoniaceae) es una especie forestal nativa del Brasil que se propaga exclusivamente por semillas. Es una especie con potencial maderero, ornamental y de recuperación de áreas marginales de bosques brasileños. Puede alcanzar 15 a $23 \mathrm{~m}$ de altura y 40 a $60 \mathrm{~cm}$ de diámetro de fuste (DAP). Zeyheria tuberculosa produce anualmente semillas, las cuales deben ser colocadas a germinar inmediatamente después de la recolección, debido a su breve viabilidad (Lorenzi 1992). La falta de información para el análisis de semillas de esta especie dificulta su aprovechamiento en programas silviculturales, siendo fundamental estudios germinativos (Ferreira 2000).

La prueba de conductividad eléctrica evalúa indirectamente el grado de estructuración de las membranas celulares, mediante la determinación de la cantidad de iones lixiviados en la solución de imbibición. Los iones lixiviados son inversamente proporcionales a la integridad 
de las membranas celulares. Las semillas se sumergen en un determinado volumen de agua, bajo temperatura controlada durante un periodo de tiempo determinado. Como consecuencia de una menor estructura y selectividad de las membranas celulares, las semillas de menor potencial fisiológico liberan mayor concentración de iones lixiviados (Marcos Filho et al. 1987).

La prueba de la conductividad eléctrica ha sido propuesta como un ensayo para evaluar el vigor de las semillas, considerando que semillas con bajo vigor generalmente presentan menor velocidad de restaurar la integridad de las membranas celulares. Esta prueba presenta la ventaja de rapidez, objetividad, bajo costo y poseer base teórica consistente, siendo capaz de identificar el deterioro de las semillas en su estado inicial (AOSA 1983, Hampton y Tekrony 1995).

La presente investigación tuvo por objetivo emplear la prueba de la conductividad eléctrica y estudiar el volumen de agua y el periodo de imbibición para la evaluación de la calidad fisiológica de de semillas de Z tuberculosa.

\section{MÉTODOS}

Los experimentos fueron realizados en el Laboratorio de Semillas Hortícolas y Forestales del Departamento de Producción Vegetal de la Universidad Estadual Paulista UNESP-FCAV, Campus de Jaboticabal, San Pablo, Brasil.

La clasificación climática para esta región donde se encuentran los árboles de Z. tuberculosa según (Köppen 1973) es de tipo Cwa, subtropical húmedo con estiaje en invierno; la precipitación y las temperaturas medias anuales se sitúan próximas de $1.400 \mathrm{~mm}$ y $21^{\circ} \mathrm{C}$, respectivamente. La humedad relativa media del aire es de $75 \%$ y la velocidad media del viento es de $5,04 \mathrm{~km} \mathrm{~h}^{-1}$.

Fueron seleccionados cuatro árboles madre (AM) de 17 años de edad, ubicados en un área de reforestación a una altitud de $612 \mathrm{~m}$ s.n.m. Los árboles madre presentan las siguientes características: buen aspecto fitosanitario, vigor (resistente a vientos fuertes y a cambios de temperatura), libre de plagas y enfermedades (observado visualmente), buena producción de semillas y árboles no aislados, conforme los criterios establecidos por (Capelanes y Biella 1984). Los árboles madre fueron referenciados geográficamente: árbol madre $1,21^{\circ} 15^{\prime} 17,53^{\prime \prime} \mathrm{S}, 48^{\circ} 17^{\prime} 01,32^{\prime \prime} \mathrm{O}$ (lote I); árbol madre 2, $21^{\circ} 15^{\prime}$ 17,63" ' S, 48 15' 17,63" O (lote II); árbol madre 3, $21^{\circ} 15^{\prime} 18,40$ " S, 48 $18^{\circ}$ ' 58,92" O (lote III); árbol madre 4, $21^{\circ} 15^{\prime}$ ' 18,95" $\mathrm{S}, 48^{\circ} 16^{\prime}$ ' 58,11 " O (lote IV).

Los frutos fueron colectados manualmente y dispuestos en bolsas plásticas e inmediatamente transportados al vivero experimental de plantas ornamentales y forestales de la UNESP-FCAV. Las semillas fueron extraídas de los frutos manualmente y secadas al sol por ocho horas y en la sombra por 24 horas. Con anterioridad a los ensayos se determinó el porcentaje de humedad de las semillas. Para este propósito se utilizó el método de estufa a $105^{\circ} \mathrm{C} \pm 3{ }^{\circ} \mathrm{C}$ por 24 horas, según criterios establecidos por el Ministério de Agricultura y Reforma Agraria (1992), utilizándose dos repeticiones de 20 semillas por lote.

La prueba de germinación fue realizada en una cámara vertical, tipo B.O.D, con ocho horas de luz/día (fotoperiodo) y con temperatura de $25{ }^{\circ} \mathrm{C}$. Para cada lote fueron usadas cinco repeticiones de 20 semillas, previamente desinfectadas por inmersión en solución de hipoclorito de sodio ( $2 \%)$ por un minuto. Posteriormente, las semillas fueron lavadas con agua desionizada. Las semillas fueron distribuidas en cajas de plástico con tapa transparente ("gerbox") de 11 x 11 x $4 \mathrm{~cm}$, sobre dos hojas de papel previamente esterilizado en estufa a $105^{\circ} \mathrm{C}$ durante 2 horas y humedecida con agua desionizada; el volumen del agua usado fue equivalente a 2,5 veces el peso del papel (Ministério da Agricultura e Reforma Agrária 1992).

El conteo de semillas germinadas fue realizado diariamente hasta que la germinación se detuvo. Las semillas se consideraron germinadas cuando la radícula alcanzó $2 \mathrm{~mm}$ de largo (Labouriau 1983).

Los datos de porcentaje de germinación e índice de velocidad de germinación (IVG) fueron analizados estadísticamente por bloques al azar, con cinco repeticiones por lote (árbol madre) y las medias fueron comparadas entre sí por la prueba de comparaciones múltiples de Tukey $(P<0,05)$.

Para la prueba de la conductividad eléctrica (CE) fueron utilizadas cinco repeticiones de 20 semillas por lote. Las semillas previamente fueron pesadas en balanza de precisión $(0,0001 \mathrm{~g})$ y posteriormente fueron colocadas para embeber en 75,100 y $125 \mathrm{~mL}$ de agua desionizada en los siguientes periodos de tiempo: 2, 4, 6, 8, 12, 24, 48 y 72 horas, a $25^{\circ} \mathrm{C}$. Después de cada período de imbibición, los valores de la conductividad eléctrica fueron medidos empleándose un conductivímetro de la marca Digimed CD-21. El valor de cada lectura de conductividad eléctrica fue dividido por el respectivo peso de la muestra y los resultados fueron expresados en $\mu \mathrm{S} \mathrm{cm}^{-1} \mathrm{~g}^{-1}$ de semilla (AOSA 1983).

Los resultados de la conductividad eléctrica fueron analizados separadamente, para cada combinación del volumen de agua por el tiempo y las medias fueron comparadas por la prueba de Tukey $(P<0,05)$. El modelo estadístico empleado fue de bloques al azar, con factores de cuatro lotes de semillas por tres volúmenes de agua y por ocho tiempos de imbibición. Para evaluar el progreso de los valores de conductividad eléctrica con el aumento de tiempo de imbibición fueron estimadas ecuaciones de regresión polinomial para cada tratamiento en función de la conductividad eléctrica, eligiéndose la ecuación de mayor grado de significancia estadística al $5 \%$ por la prueba F.

\section{RESULTADOS}

Se observó una pequeña variación en el contenido de agua en los lotes de semillas. El lote II superó los demás 
lotes de semillas en cuanto a porcentaje de germinación, seguido del lote I. El lote III fue inferior a los lotes I y II; el lote IV presentó el menor porcentaje de germinación. Los coeficientes de variación experimental variaron de medios a muy altos, de acuerdo con la clasificación de Gomes (2000), en donde los valores ubicados entre 0 y 10 son considerados bajos, de 10 a 20 son medios y sobre 20 son muy altos (cuadro 1).

Se observó una adecuada selección de semillas en los cuatro árboles madre por los resultados de los análisis estadísticos referentes a los valores de la conductividad eléctrica que juzgaron los lotes de forma semejante según la prueba de germinación (cuadro 1 y figura 1). Muchas veces esta relación no ocurre, muchas veces la prueba de germinación revela una información diferente a la prueba de vigor empleada.

Se verificó que el lote II obtuvo los valores de conductividad eléctrica más bajos (figura 1) en todos los períodos de imbibición. Le siguen el lote III y el lote I. El lote IV alcanzó la mayor cantidad de iones lixiviados, considerado como de bajo vigor. Los resultados indican que el lote II presentó alto vigor, pues para todos los períodos de imbibición éste se destacó con bajos valores de conductividad eléctrica (figura 1). Estos resultados son coherentes con la prueba de germinación (cuadro 1). De forma general, los resultados obtenidos indican un aumento progresivo de los lixiviados con el transcurrir de cada período de imbibición.

Comparando los volúmenes de agua de 75, 100 y $125 \mathrm{~mL}$ (figura 1), las semillas del lote IV fueron las que lixiviaron más iones en comparación con los demás lotes de semillas. Ello incrementó con el aumento del tiempo de imbibición.

Según los tres volúmenes de agua experimentados, hubo una mejor selección de los lotes a partir de 12 horas de imbibición, constatándose un distanciamiento de las curvas de conductividad eléctrica en los lotes I, II y III en comparación con el lote IV (figura 1). Se observó aumento en los valores de la conductividad eléctrica a medida que se prolongó el tiempo de imbibición, permitiendo una diferenciación más nítida entre los lotes, de esa forma posibilitó una buena selección o discriminación de los mismos.

\section{DISCUSIÓN}

La prueba de la conductividad eléctrica demostró ser adecuada para la selección de lotes de semillas de Zeyheria tuberculosa con diferente potencial fisiológico y ella puede ser realizada a $25{ }^{\circ} \mathrm{C}$ de temperatura, con el uso de cuatro repeticiones de 25 semillas en 75 o $125 \mathrm{~mL}$ de agua desionizada, y la lectura de la conductividad eléctrica puede ser realizada al finalizar 24 horas de imbibición.

El bajo contenido de agua obtenido en los lotes de semillas en relación al sugerido (cuadro 1), no afectó los resultados de la prueba de la conductividad eléctrica como lo observado por Marques et al. (2002b). Los valores de coeficientes de variación obtenidos en el presente estudio son medios a altos (Gomes 2000). Estos, a su vez, son comparables con resultados de experimentos con semillas forestales nativas de árboles no mejorados genéticamente, indicando una alta variabilidad entre individuos.

En el proceso de la conductividad eléctrica, la cantidad de exudados liberados por las semillas vigorosas va estabilizándose debido a la reorganización de las membranas (Rosa et al. 2000). En este estudio se pudo observar este fenómeno en los lotes I, II y II de semillas considerados vigorosos (figura 1).

En la presente investigación se obtuvieron excelentes resultados, tanto para la prueba de la conductividad eléctrica como para la prueba de germinación. Lo ideal es que ambas pruebas mantengan estrecha relación como constatado por Santos (2004). Los lotes presentaron semillas en diferentes niveles de vigor (cuadros 1 y figura 1), sugiriéndose su uso de esta prueba de conductividad eléctrica para estudiar las semillas de esta especie. Cabe destacar que no siempre la conductividad eléctrica acompañada con la prueba de germinación son adecuadas en la diferenciación de la calidad fisiológica de diferentes lotes de

Cuadro 1. Contenido de agua (CA, gramos), porcentaje de semillas germinadas (\% G), índice de velocidad de germinación (IVG) y masa de materia seca de plántulas (MSP, g plántula ${ }^{-1}$ ) provenientes de cuatro lotes de semillas de Zeyheria tuberculosa.

Water content (CA), percentage of seed germination (\% G), speed of germination index (IVG), dry matter seedlings (MSP) obtained of four lots of seed Zeyheria tuberculosa.

\begin{tabular}{lcccc}
\hline Lote de semillas & $\mathrm{CA}(\mathrm{g})$ & $\% \mathrm{G}$ & $\mathrm{IVG}$ & MSP \\
\hline lote I & 8,14 & $82 \mathrm{a}$ & $1,66 \mathrm{ab}$ & $0,026 \mathrm{~b}$ \\
lote II & 8,06 & $92 \mathrm{a}$ & $1,84 \mathrm{a}$ & $0,037 \mathrm{a}$ \\
lote III & 7,49 & $59 \mathrm{~b}$ & $1,33 \mathrm{~b}$ & $0,026 \mathrm{~b}$ \\
lote IV & 10,09 & $19 \mathrm{c}$ & $0,53 \mathrm{c}$ & $0,017 \mathrm{c}$ \\
Media & - & 63 & 1,34 & 0,029 \\
CV $(\%)$ & - & 19,6 & 25,0 & 27,8 \\
\hline
\end{tabular}

Medias seguidas por una misma letra no difieren por la prueba de Tukey $(P>0,05)$. 
BOSQUE 32(2): 197-202, 2011

Pruebas de vigor en semillas de Zeyheria tuberculosa

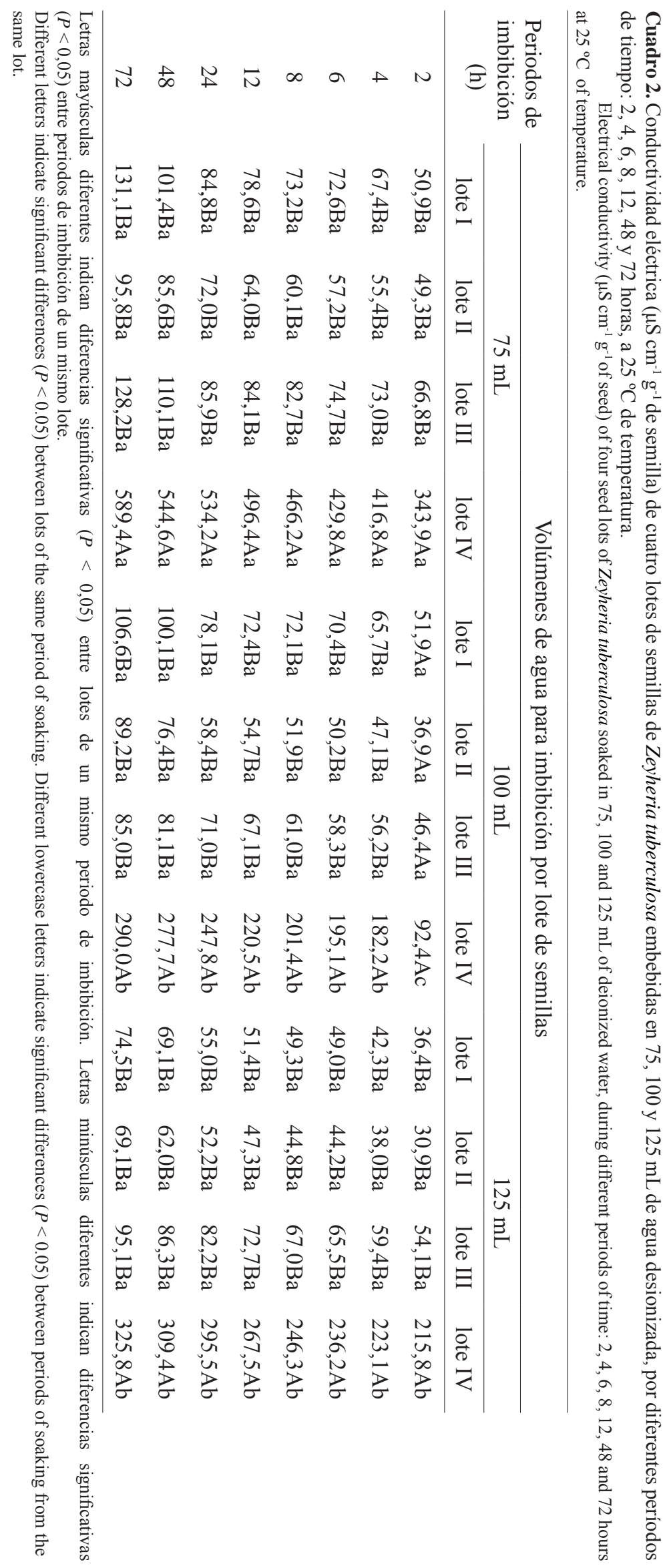




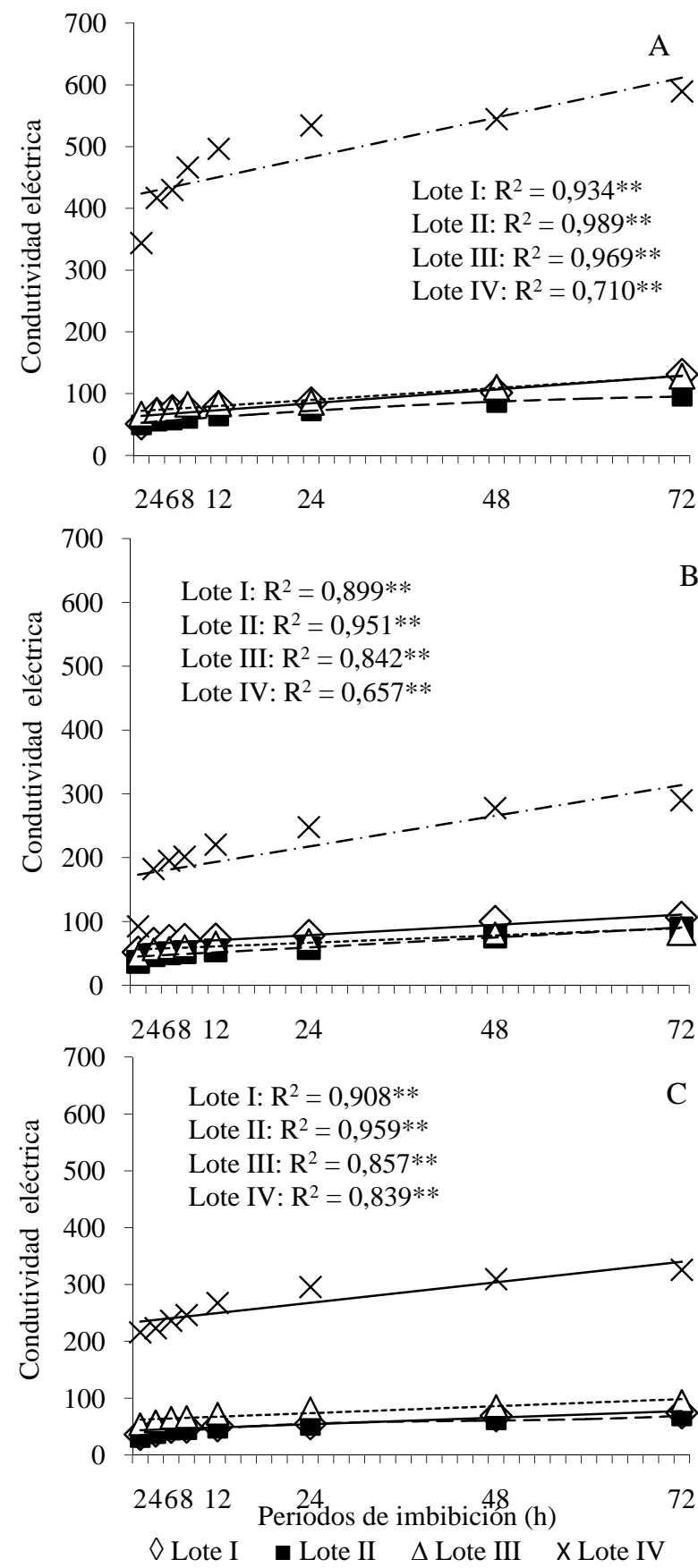

Figura 1. Conductividad eléctrica $\left(\mu \mathrm{S} \mathrm{cm}^{-1} \mathrm{~g}^{-1}\right)$ de semillas de Zeyheria tuberculosa provenientes de cuatro lotes de semillas y embebidas en agua desionizada por diferentes períodos de imbibición (2, 4, 6, 8, 12, 24, 48 y 72 horas) en $75 \mathrm{~mL}$ (A), $100 \mathrm{~mL}$ (B) y $125 \mathrm{~mL}$ (C). A $25^{\circ} \mathrm{C}$ de temperatura.

Electrical conductivity $\left(\mu \mathrm{S} \mathrm{cm}^{-1} \mathrm{~g}^{-1}\right)$ of seeds Zeyheria tuberculosa from four lots seeds and imbibitions at different imbibitions periods (2, 4, 6, 8, 12, 24, 48 and 72 hours) in $75 \mathrm{~mL}$ (A), $100 \mathrm{~mL}$ (B) and $125 \mathrm{~mL}$ (C) of deionized water, at $25^{\circ} \mathrm{C}$ of temperate. semillas. Factores como la variabilidad genética existente dentro de la especie pueden influir en el resultado (Soto et al. 2009). Para Leonhardt (2000) la conductividad eléctrica no fue eficiente para identificar el período de madurez fisiológica de Citharexylum montevidense (Spreng.) Mold, cuando la determinó a $20^{\circ} \mathrm{C}$ en ocho repeticiones de 20 semillas en $100 \mathrm{~mL}$ de agua por un período de ocho horas. Por otra parte, Santos (2004) verificó que ambas pruebas de conductividad eléctrica y de germinación posibilitaron igualmente la selección de lotes de semillas de Sebastiania commersoniana (Baill.) Smith et Downs., resaltando que la estandarización y repetibilidad de la mayoría de las pruebas de vigor son más difíciles que para la prueba de germinación.

Determinar la calidad fisiológica de lotes de semillas de una especie en un periodo de corto tiempo se torna práctico y económico para pruebas de vigor en condiciones de laboratorio y tiene ventajas de facilitar la selección entre lotes de semillas con diferente potencial fisiológico, ya sea para comercialización, producción de plantas vigorosas o simplemente cuando son usadas en proyectos de gestión de bosques.

\section{REFERENCIAS}

AOSA (Association of Official Seed Analysts, US). 1983. Seed vigour testing handbook. Lincoln, USA. AOSA. 88 p.

Capelanes TMC, Biella LC. 1984. Programa de produção e tecnologia de sementes de espécies florestais nativas desenvolvido pela Companhia Energética de São Paulo - CESP. In Simpósio brasileiro sobre tecnologia de sementes florestais. Belo Horizonte, Brasil. p. 85-107.

Ferreira CAC. 2000. Recuperação de áreas degradadas. Informe Agropecuário 21(202): 127-130.

Gomes FP. 2000. Curso de estatística experimental. 10 ed. Piracicaba, San Pablo, Brasil. Nobel. 468 p.

Hampton JG, DM Tekrony. 1995. Handbook of vigour test methods. 3 ed. Zurich, Suiza. The International Seed Testing Association. $117 \mathrm{p}$.

Köppen W. 1973. Das geographische System der Klimate. Berlin. Gebruder Borntraeger. 369 p.

Labouriau LG. 1983. A germinação das sementes. Washington, USA. OEA. 174 p.

Leonhardt C. 2000. Maturação fisiológica de sementes de tarumã-de-espinho (Cintharexylum montevidense (Spreng) Moldenke - Verbenaceae). Tesis de Maestría en Ciencia y Tecnología de Semillas. Pelotas, Brasil. Facultad de Agronomía "Eliseu Maciel" Universidad Federal de Pelotas. 25p.

Lorenzi H. 1992. Árvores brasileiras: Manual de identificação e cultivo de plantas arbóreas nativas do Brasil. Nova Odessa, Brasil. Editora Plantarum. 368 p.

Marcos Filho J, SM Cícero, WR Silva. 1987. Avaliação da qualidade das sementes. Piracicaba, San Pablo. Brasil. Fealq. $230 \mathrm{p}$.

Marques MA, RC Paula, TJD Rodrigues. 2002b. Efeito do número de sementes e do volume de água na condutividade elétrica de sementes de Dalbergia nigra (Vell.) Fr. All. ex. Benth. Revista Brasileira de Sementes 24(1): 254-262. 
Ministério da Agricultura e Reforma Agrária. 1992. Regras para analise de sementes. Brasília, Brasil. 356 p.

Rosa SDVF, EVRV Pinho, MGGC Vieira, RD Veiga. 2000. Eficácia do teste de condutividade elétrica para uso em estudos de danos de secagem em sementes de milho. Revista Brasileira de Sementes 22(1): 54-63.

Santos SRG. 2004. Qualidade fisiológica e armazenamento de sementes de Sebastiania commersoniana (Baill.) Smith and Downs. 2004. Tesis Doctorado en Agronomía Producción Vegetal, Jaboticabal, Brasil. Facultad de Ciencias Agrarias y Veterinarias, Universidad Estadual Paulista. 95 p.

Soto GJL, RC Paula, SV Valeri. 2009. Teste de condutividade elétrica em sementes de Albizia hassleri (Chodat) Burkart. Fabaceae-mimosoideae. Revista Árvore 33(4): 625-634.

Recibido: 08.11.10

Aceptado: 20.05.11 